\title{
The Origin of Large-Scale Magnetic Fields in Low-Mass Galaxies
}

\author{
Prasanta Bera $1,2,3, *,+$ (i) , Anvar Shukurov $4, *,+$ (i) and Kandaswamy Subramanian $1,+(1)$ \\ 1 Inter-University Centre for Astronomy and Astrophysics, Post Bag 4, Pune 411 007, India; kandu@iucaa.in \\ National Centre for Radio Astrophysics, TIFR, Post Bag 3, Pune 411 007, India \\ Mathematical Sciences and STAG Research Centre, University of Southampton, Southampton SO17 1BJ, UK \\ School of Mathematics, Statistics and Physics, Newcastle University, Newcastle upon Tyne NE1 7RU, UK \\ * Correspondence: pbera.phy@gmail.com (P.B.); anvar.shukurov@ncl.ac.uk (A.S.) \\ + These authors contributed equally to this work.
}

Received: 14 October 2019; Accepted: 26 November 2019; Published: 29 November 2019

\begin{abstract}
The origin of large-scale magnetic fields, detected in some low-mass (dwarf and irregular) galaxies via polarised synchrotron emission and Faraday rotation, has remained unexplained for a long time. We suggest that mean-field dynamos can be active in galaxies of this class despite their slow rotation because their discs are relatively thick. Earlier assessments of the possibility of the mean-field dynamo action in low-mass galaxies relied on estimates applicable to thin discs, such as those in massive spiral galaxies. Using both order-of-magnitude estimates and numerical solutions, we show that the strength of differential rotation required to amplify magnetic field reduces as the aspect ratio of the galactic gas layer increases. As in a thin disc, quadrupolar magnetic fields dominate in thick discs. Thus, the origin of large-scale magnetic fields in low-mass galaxies has been clarified. This class of galaxies provides a new ground for testing our understanding of galactic magnetism.
\end{abstract}

Keywords: dwarf galaxies; irregular galaxies; interstellar medium; magnetic fields; dynamo; radio continuum

\section{Introduction}

Large-scale magnetic fields are a prominent and widespread in disc galaxies [1-3]. Their origin is most likely due to the mean-field dynamo action that relies on random (turbulent) motions of the partially ionised interstellar gas, galactic differential rotation, and stratification of the gas distribution in the galactic gravity field. The mean-field dynamo theory (for a review, see [4]) explains many salient properties of galactic magnetic fields [5] (for a compendium of basic results, see [6]). Galactic dynamo theory has been applied, with a significant success, to a number of specific spiral galaxies, including the Milky Way, M31, M33, M51 [7], and a sample of barred galaxies ([8] and references therein). Statistical comparisons of theory and observations of galactic magnetic fields can be found in Van Eck et al. [9] and Chamandy et al. [10]. Rodrigues et al. [11,12] discuss galactic mean-field dynamos in the context of galaxy formation theory.

Applications of the mean-field dynamo theory to galaxies are facilitated by the fact that the discs of spiral galaxies are thin (beyond a few hundred parsecs from the rotational axis) as the ratio of their scale height to radius is much less than unity, $\epsilon=h / r \ll 1$. Near the Sun, this ratio is about 0.06 for the warm interstellar gas ( $h \simeq 0.5 \mathrm{kpc}$ ) and the galactocentric distance $r=8.5 \mathrm{kpc}$. Asymptotic expansion in $\epsilon$ reduces the mean-field dynamo equations to tractable forms that can be efficiently analysed using both analytical and numerical methods. 
Dynamo action is a threshold phenomenon: for a magnetic field to be sustained by plasma motions, the strength of magnetic induction effects due to large-scale and turbulent flows has to exceed the intensity of dissipation. In the case of a mean (large-scale) magnetic field, the main dissipation mechanism is the turbulent magnetic diffusion. The intensity of the mean-field dynamo action is measured by the dynamo number $D$ that can be expressed (in a simplified form) in terms of observable quantities as (for details, see [1])

$$
D=\frac{\alpha_{0} \Omega S h^{3}}{\beta^{2}} \simeq 9 \frac{h^{2} \Omega S}{v_{0}^{2}},
$$

where $\alpha_{0} \simeq l_{0}^{2} \Omega / h$ is a measure of the mean helicity of the turbulent motions; $\Omega$ is the angular velocity of galactic rotation; $S=r \partial \Omega / \partial r$ is the shear rate of the rotation ( $r$ is the galactocentric distance); $h$ is the scale height of the gas disc, $\beta \simeq \frac{1}{3} l_{0} v_{0}$ is the turbulent magnetic diffusivity; and $l_{0}$ and $v_{0}$ are the turbulent scale and root-mean-square speed. (We note that $D$ is negative in the main parts of galaxies since $\partial \Omega / \partial r<0$, so $S<0$.) A central result of the theory of mean-field dynamo in a thin disc is the marginal value of the dynamo number required for the dynamo action, $D_{\mathrm{c}} \approx-10$ : the magnetic field can grow and be maintained by interstellar turbulence and rotation when the magnitude of the dynamo number is large enough, $|D|>\left|D_{\mathrm{c}}\right|$, and it decays otherwise. We stress-this will be essential later-that this value of $D_{\mathrm{c}}$ only applies to a thin disc, $\epsilon=h / r \ll 1$.

Despite successes of the galactic dynamo theory, there is a class of galaxies whose large-scale magnetic fields were hitherto unexplainable; namely, the low-mass (dwarf and irregular) galaxies. In some but not all nearby dwarf galaxies, radio observations of synchrotron polarisation and Faraday rotation have revealed magnetic fields ordered on scales comparable to the galaxy size and exceeding the turbulent scales. Their strength is similar to, albeit often weaker than, that in normal galaxies, e.g., in NGC 4449 [13], the Large and Small Magellanic Clouds [14-16] and NGC 1569 [17]. Chyży et al. [18] detected synchrotron emission indicating random magnetic fields in three (IC 10, NGC 6822, and IC 1613) out of twelve Local Group irregular and dwarf irregular galaxies, with the upper limits of 3-5 $\mu \mathrm{G}$ for the total magnetic fields in the remaining cases. Hindson et al. [19] detected radio emission at $\lambda 6 \mathrm{~cm}$ from 22 out of 40 nearby dwarf galaxies to find the average strength of the total magnetic field of 5-8 $\mu \mathrm{G}$ (comprised of both random and possible large-scale parts) assuming energy equipartition between cosmic rays and magnetic fields. Rodrigues et al. [12] found that $20 \%-50 \%$ of low-mass galaxies might host detectable large-scale magnetic fields, in agreement with (see arXiv:1302.5663 for an up-to-date list of galaxies) Beck and Wielebinski [20].

In comparison with large spiral galaxies, dwarf and irregular galaxies have lower masses and correspondingly shallower gravitational potential wells. As a result, they have lower rotation speeds of the order of $50-100 \mathrm{~km} \mathrm{~s}^{-1}$ or less, but a similarly intense turbulence (see, e.g., Table 3 in [18] and references therein). For example, the maximum rotation speeds of IC 2574 and IC 10 are about $66 \mathrm{~km} \mathrm{~s}^{-1}$ and $40 \mathrm{~km} \mathrm{~s}^{-1}$, respectively [21,22]. Rotation is often closer to solid-body than in spiral galaxies [23,24]; as an extreme example, the maximum rotation speed in IC 2574 is reached at the galactocentric distance of about $8 \mathrm{kpc}[21,25]$, suggesting a typical angular velocity $\Omega \lesssim 10 \mathrm{~km} \mathrm{~s}^{-1} \mathrm{kpc}^{-1}$. Typical mean circular velocities in a sample of $17 \mathrm{dwarf}$ irregular galaxies [24] range from 16 to $63 \mathrm{~km} \mathrm{~s}^{-1}$; their HI velocity dispersion is $8-20 \mathrm{~km} \mathrm{~s}^{-1}$; and the radius of the observable HI disc is $0.4-6.4 \mathrm{kpc}$. The 26 dwarf galaxies observed in HI by $\mathrm{Oh}$ et al. [23] have maximum rotational velocities of $12-126 \mathrm{~km} \mathrm{~s}^{-1}$ reached at galactocentric distances $0.3-10 \mathrm{kpc}$.

Residing in a shallower gravitational potential, the gas discs of dwarf galaxies are 3-4 times thicker than in massive spirals. In IC 2574, the HI scale height is estimated as $h_{\mathrm{HI}} \simeq 350 \mathrm{pc}$ (and the HI volume density of $0.15 \mathrm{~cm}^{-3}$ ), whereas the one-dimensional velocity dispersion is $7 \mathrm{~km} \mathrm{~s}^{-1}$. Similarly, $h_{\mathrm{HI}} \simeq 625 \mathrm{pc}$ in Ho II, and $h_{\mathrm{HI}} \simeq 460 \mathrm{pc}$ in NGC 5023 [21]. The aspect ratio of the neutral hydrogen discs of dwarf galaxies is about 10:1 as opposed to 100:1 in massive disc galaxies [21]. The mean Hi axial ratio among 36 
low surface brightness and blue compact galaxies is 0.58 [26] - these are not flat objects. Roychowdhury et al. [27] estimated the axial ratio to be about $\epsilon=0.5$ for the faintest dwarf galaxies. As in spiral galaxies, their gas discs are flared at galactocentric distances exceeding $(3-4) R_{\star}$, where $R_{\star}$ is the radial scale length of the stellar disc: $R_{\star}=0.8,1.2,2.1$, and $0.5 \mathrm{kpc}$ in the four dwarf galaxies modelled by Banerjee et al. [28], DDO 154, Ho II, IC 2574 and NGC 2366, respectively.

Because of the slow rotation, the typical magnitude of the dynamo number (1) in the low-mass galaxies is of order unity,

$$
D \simeq 2\left(\frac{\Omega S}{100 \mathrm{~km}^{2} \mathrm{~s}^{-2} \mathrm{kpc}^{-2}}\right)\left(\frac{h}{0.5 \mathrm{kpc}}\right)^{2}\left(\frac{v_{0}}{10 \mathrm{~km} \mathrm{~s}^{-1}}\right)^{-2},
$$

significantly smaller by magnitude than $\left|D_{\mathrm{c}}\right| \approx 10$ required for the mean-field dynamo action in a thin gas layer. This has prompted suggestions that magnetic fields observed in dwarf and irregular galaxies are due to the fluctuation dynamo action that does not require any overall rotation and generates random magnetic fields with vanishing mean $[4,7,29]$. To explain the polarisation of observed radio emission, it is further suggested that differential rotation makes the random field anisotropic. However, the rotational velocity shear $S$ is also weak in low-mass galaxies, and such an option does not appear appealing. Moss and Sokoloff [30] suggest that random magnetic fields generated by the fluctuation dynamo in the disc of a dwarf galaxy can be transported by a galactic outflow to the halo where their scale increases as the gas expands. As the random magnetic fields decay, those of a larger scale survive for longer, and the scale of the magnetic field increases as it decays. However, the scale of the random magnetic field in the halo remains much smaller than the size of the galaxy (especially at smaller distances from the disc where the advected random magnetic field has not yet decayed significantly) and this process can hardly explain the polarisation patterns observed to be ordered over several kiloparsecs. Such patterns occur in low-mass galaxies observed both face-on and edge-on, suggesting that the polarised emission also originates in the disc rather than in the halo alone. There is little doubt that at least some low-mass galaxies host genuine mean magnetic fields that produce significant Faraday rotation. As in other galaxies, primordial magnetic fields are not a viable option because of the high intensity of turbulent motions.

The dominant dissipation mechanism of the large-scale magnetic field in a thin layer is turbulent diffusion across it, $\tau_{\mathrm{d}} \simeq h^{2} / \beta$, where $\beta \simeq \frac{1}{3} l_{0} v_{0}$ is the turbulent magnetic diffusivity, $l_{0}$ is the turbulent scale, and $h$ is the half-thickness (scale height) of the warm ionised gas layer. In spiral galaxies with $h \simeq 0.5 \mathrm{kpc}$ and $\beta \simeq 10^{26} \mathrm{~cm}^{2} \mathrm{~s}^{-1}$, we have $\tau_{\mathrm{d}} \simeq 5 \times 10^{8} \mathrm{yr}$. This time scale is larger in a thick disc compared to a thin disk, which reduces the relative importance of dissipation compared to the magnetic induction effects, and the mean-fled dynamo is easier to excite in a thick layer than in a thin one. The radial diffusion also becomes more important in a thick disk, and then the magnitude of the critical dynamo number $D_{\mathrm{c}}$ increases with the layer thickness. However, the dynamo number increases even faster, so that the ratio $D / D_{\mathrm{c}}$, a measure of the dynamo efficiency, is higher in a thicker disc (with all other parameters being the same). Similarly to this tendency, the dynamo efficiency in spheroids increases along the sequence from a flattened spheroid to the sphere [31]. Stix [32], Brandenburg et al. [33], and Elstner et al. [34] also show that mean-field dynamos are more efficient in thicker discs and oblate spheroids, and that magnetic fields of the quadrupolar symmetry are generated at smaller dynamo numbers than the dipolar fields.

Siejkowski et al. [35] (see also [36,37]) present numerical simulations of the mean-field dynamo action in low-mass galaxies but adopt turbulent magnetic diffusivity of $\beta=3 \times 10^{25} \mathrm{~cm}^{2} \mathrm{~s}^{-1}$, three times lower than the more plausible value of $\beta=10^{26} \mathrm{~cm}^{2} \mathrm{~s}^{-1}$. The spatial resolution of the simulations, $47 \mathrm{or} 78 \mathrm{pc}$, is close to the turbulent scale, so that turbulent motions are not resolved and the adopted value of $\beta$ is likely to be close to the effective one in the simulations. As shown in Equation (1), the dynamo number in those 
simulations can, thus, be overestimated by a factor $\approx 10$ and the efficiency of the dynamo action appears to be exaggerated, which makes the significance of these results questionable.

In this paper, we discuss solutions of the mean-field dynamo equation in thick discs to demonstrate that it can explain the amplification and maintenance of large-scale magnetic fields in low-mass galaxies. Having presented basic equations in Section 2, we discuss dynamo action in a thick disc in Section 3 where the value of $D_{\mathrm{c}}$ is estimated and the corresponding minimum rotational speed required for the dynamo action in a thick disc is derived. Numerical solutions of the dynamo equation are presented in Section 4 and explored in Section 5 with emphasis on the dependence of $D_{\mathrm{c}}$ and the corresponding critical rotational speed on the disc aspect ratio; the accuracy of the estimates of Section 3 is confirmed. The results are discussed and summarised in Section 6.

\section{Basic Equations}

The generation of a mean (large-scale) magnetic field $\boldsymbol{B}$ by a random velocity field is governed by the mean-field dynamo equation [38-41]

$$
\frac{\partial \boldsymbol{B}}{\partial t}=\nabla \times[\boldsymbol{V} \times \boldsymbol{B}+\alpha \boldsymbol{B}-\beta \nabla \times \boldsymbol{B}]
$$

where $V=\Omega \times r$ is the large-scale velocity dominated by rotation with the angular velocity $\Omega$ and the effects of galactic random flows (turbulence) are represented by the turbulent transport coefficients $\alpha=-\frac{1}{3} \tau\langle\boldsymbol{v} \cdot(\nabla \times v)\rangle \simeq l_{0}^{2} \Omega / h$ (where $l_{0}$ is the turbulent scale and $h$ is the density scale height), which depends on the mean helicity of the turbulence $\langle v \cdot(\nabla \times v)\rangle$, and turbulent diffusivity $\beta \simeq \frac{1}{3} \tau\left\langle v^{2}\right\rangle$ (assumed to be much larger than the Ohmic magnetic diffusivity), with $v$ the turbulent velocity field, $\tau$, its correlation time and angular brackets denote an appropriate average over the turbulent fluctuations.

We model a galaxy as an axisymmetric disc with a Brandt rotation curve $V(r)=r \Omega(r)$,

$$
\Omega(r)=\frac{V_{0}}{R_{0}}\left[\frac{1}{3}+\frac{2}{3}\left(\frac{r}{R_{0}}\right)^{n}\right]^{-3 /(2 n)},
$$

in terms of cylindrical polar coordinates $(r, \phi, z)$, with $n=2$, where $V_{0}$ and $R_{0}$ are the characteristic velocity and radial scale length. The corresponding velocity shear rate is defined as $S=r \partial \Omega / \partial r$. The half-thickness (or scale height) of the ionised gas disc is denoted $h$. For the interstellar turbulence, we assume that the correlation scale, $l_{0}=0.1 \mathrm{kpc}$ and speed $v_{0}=10 \mathrm{~km} \mathrm{~s}^{-1}$, are both similar to those in spiral galaxies.

We consider the simplest axisymmetric solutions to the mean-field equation, and so the mean magnetic field can be written as

$$
\boldsymbol{B}=B_{\phi}(r, z) \hat{\boldsymbol{\phi}}+\nabla \times\left[A_{\phi}(r, z) \hat{\boldsymbol{\phi}}\right],
$$

where $B_{\phi}$ and $A_{\phi}$ are the azimuthal components of the magnetic field and the vector potential, respectively, and $\hat{\phi}$ is the unit azimuthal vector. The assumption of axial symmetry may be rather crude when applied to low-mass galaxies but it is sufficient to assess the possibility of the mean-field dynamo action, our main goal in this paper. More realistic non-axisymmetric solutions and non-axisymmetric galaxies will be discussed elsewhere.

We use dimensionless variables, here denoted with tilde, defined in terms of the characteristic values denoted with subscript zero:

$$
\begin{aligned}
r & =R_{0} \tilde{r}, \quad z=h_{0} \tilde{z}, \quad t=\left(h_{0}^{2} / \beta\right) \tilde{t}, \quad B_{\phi}=B_{0} \tilde{B}_{\phi}, \\
A_{\phi} & =h_{0} B_{0} \tilde{A}_{\phi}, \quad \Omega=\Omega_{0} \tilde{\Omega}, \quad S=S_{0} \tilde{S}, \quad S_{0}=\Omega_{0}, \quad \alpha=\alpha_{0} \tilde{\alpha},
\end{aligned}
$$


where

$$
\alpha_{0}=l_{0}^{2} \Omega_{0} / h_{0}, \quad \Omega_{0}=V_{0} / R_{0},
$$

and we drop tilde at the dimensionless variables unless specified otherwise or obvious from the context. Since we here only consider kinematic dynamo solutions that vary exponentially in time, the characteristic magnetic field $B_{0}$ can be chosen arbitrarily and can be used to normalise the results as convenient.

We consider a flat gas layer with $h=h_{0}=$ const. Disc flaring can easily be included [1] but it does not change our conclusions regarding the possibility of dynamo action at relatively small galactocentric distances although the radial distribution of magnetic field can be affected (e.g., [42]). The coefficient $\alpha(r, z)$ is assumed to be factorisable: $\alpha=\alpha_{0} \alpha_{1}(z) \Omega(r)$ since $\alpha \propto \Omega(r)$, where we adopt $\alpha_{1}(z)=\sin (\pi z / h)$ for numerical solutions; this choice affects the results only weakly.

In terms of the dimensionless variables, the cylindrical components of the dynamo equation reduce to

$$
\begin{aligned}
\frac{\partial B_{\phi}}{\partial t} & =-R_{\omega} S \frac{\partial A_{\phi}}{\partial z}-R_{\alpha} \frac{\partial}{\partial z}\left(\alpha \frac{\partial A_{\phi}}{\partial z}\right)+\frac{\partial^{2} B_{\phi}}{\partial z^{2}}+\epsilon^{2} \mathcal{L}_{r}\left(B_{\phi}\right), \\
\frac{\partial A_{\phi}}{\partial t} & =R_{\alpha} \alpha B_{\phi}+\frac{\partial^{2} A_{\phi}}{\partial z^{2}}+\epsilon^{2} \mathcal{L}_{r}\left(A_{\phi}\right)
\end{aligned}
$$

where

$$
R_{\alpha}=\frac{h_{0} \alpha_{0}}{\beta}, \quad R_{\omega}=\frac{S_{0} h^{2}}{\beta}, \quad \epsilon=\frac{h_{0}}{R_{0}}, \quad \mathcal{L}_{r}(F)=\frac{\partial}{\partial r}\left[\frac{1}{r} \frac{\partial(r F)}{\partial r}\right] .
$$

and we note that $\alpha(r, z)$ and $S(r)$ are functions of position. Magnetic induction effects are quantified by dimensionless dynamo numbers $R_{\omega}$ for the differential rotation and $R_{\alpha}$ for the helical turbulence. The dynamo system is controlled by three dimensionless parameters, $\epsilon, V_{0} / v_{0}$, and $l_{0} / h_{0}$ via

$$
R_{\alpha}=3 \epsilon \frac{l_{0}}{h_{0}} \frac{V_{0}}{v_{0}}, \quad R_{\omega}=3 \epsilon \frac{h_{0}}{l_{0}} \frac{V_{0}}{v_{0}} .
$$

An advantage of selecting $R_{0}$ (the radial scale of the rotation curve) as the unit radial length is that the estimates and numerical results presented below are insensitive to the specific value of $R_{0}$, as this parameter enters the results only via the disc aspect ratio $\epsilon=h_{0} / R_{0}$.

When the term with $R_{\alpha}$ in Equation (8) is neglected, the dynamo system is called the $\alpha \omega$-dynamo and its efficiency is fully characterised by the single dimensionless number, the dynamo number

$$
D=-R_{\alpha} R_{\omega}=-9 \epsilon^{2} \frac{V_{0}^{2}}{v_{0}^{2}}
$$

This approximation is appropriate when the differential rotation is sufficiently strong to have $R_{\omega} \gg$ $R_{\alpha}$. Otherwise, the dynamo is known as the $\alpha^{2} \omega$-dynamo, characterised by two dimensionless numbers, $R_{\alpha}$ and $R_{\omega}$, or, equivalently, $R_{\alpha}^{2}$ and $D$. For parameter values typical of low-mass galaxies, $\epsilon=0.5$, $V_{0} / v_{0}=5-10$ and $l_{0} / h_{0}=0.2$, we get $R_{\alpha}=1.5-3$ and $R_{\omega} \simeq 30-70$.

\section{Mean-Field Dynamo in a Thick Disc}

As any other dynamo mechanism, the mean-field dynamo is a threshold phenomenon: the mean magnetic field can be amplified and then maintained against turbulent diffusion only if the induction effects are strong enough. When differential rotation is strong (the $\alpha \omega$-dynamo approximation), the mean magnetic field grows and then reaches a steady state if $|D|>\left|D_{\mathrm{c}}\right|$ for a certain $D_{\mathrm{c}}$ and decays if $|D|<\left|D_{\mathrm{c}}\right|$. 
In galaxies with significant differential rotation, it dominates over the $\alpha$-effect in Equation (8) and the term with $R_{\alpha}$ can be neglected in this equation. To obtain an estimate of the dynamo threshold in a thick disc of a dwarf galaxy, we note that, in terms of dimensionless variables, spatial derivatives in Equations (8) and (9) are of order unity in $\epsilon$. In the spirit of the no- $z$ approximation introduced by Subramanian and Mestel [43] and refined by Phillips [44] (details and further justification can be found in [45]), we get the following estimates: $\left|\partial B_{\phi} / \partial z\right| \simeq \pi\left|B_{\phi}\right| / 2,\left|\partial^{2} B_{\phi} / \partial z^{2}\right| \simeq-\pi^{2}\left|B_{\phi}\right| / 4$ and $\left|\mathcal{L}_{r}\left(B_{\phi}\right)\right| \simeq-k^{2}\left|B_{\phi}\right|$, and similar ones for the derivatives of $A_{\phi}$. The negative sign in the second derivatives follows from the properties of the diffusion operator and $k$ is a constant of order unity.

The dynamo is called marginal when $\partial B / \partial t=\mathbf{0}$; this state corresponds to the marginal value of the dynamo number $D_{\mathrm{c}}=R_{\alpha} R_{\omega} \bar{\alpha} \bar{S}$, where $\bar{\alpha}$ and $\bar{S}$ are the values of $\alpha$ and $S$ in the radius, averaged (note that $\bar{S}<0$; hence, $D_{\mathrm{c}}<0$, and both $\bar{\alpha}$ and $\bar{\Omega}$ are dimensionless, so of order unity). For the marginal state, Equations (8) and (9) reduce, by the order of magnitude in $\epsilon$, to

$$
\frac{\pi}{2} R_{\omega, \mathrm{c}} \bar{S} A_{\phi}+\left(\frac{\pi^{2}}{4}+k^{2} \epsilon^{2}\right) B_{\phi} \simeq 0, \quad\left(\frac{\pi^{2}}{4}+k^{2} \epsilon^{2}\right) A_{\phi}-R_{\alpha, c} \bar{\alpha} B_{\phi} \simeq 0,
$$

leading to an order of magnitude estimate

$$
D_{\mathrm{c}} \simeq-\frac{2}{\pi}\left(\frac{\pi^{2}}{4}+k^{2} \epsilon^{2}\right)^{2}
$$

where we adopt $\bar{\alpha}=\bar{\Omega}=1$. Combining the estimate of $D_{\mathrm{c}}$ with Equation (12), we obtain a measure of the local dynamo efficiency as

$$
\frac{D}{D_{\mathrm{c}}} \simeq \frac{9 \pi}{2} \frac{V_{0}^{2}}{v_{0}^{2}} \frac{\epsilon^{2}}{\left(\pi^{2} / 4+k^{2} \epsilon^{2}\right)^{2}}
$$

confirming that the mean-field dynamo is more efficient in a thicker disc: this ratio increases with $\epsilon$ for $\epsilon<\pi /(2 k)$, with the right-had side smaller than unity for $k>\pi / 2$.

This estimate can also be rewritten in terms of the rotational velocity: magnetic field can be maintained when the characteristic rotation speed $V_{0}$ exceeds a critical value $V_{\mathrm{c}}$ given by

$$
V_{\mathrm{c}} \simeq v_{0} \sqrt{\frac{2}{\pi}} \frac{\pi^{2} / 4+k^{2} \epsilon^{2}}{3 \epsilon},
$$

and $V_{\mathrm{c}}$ decreases with $\epsilon$ for $\epsilon<\pi /(2 k)$. This confirms again that the mean-field dynamo is easier to excite in a thicker disc.

It is convenient to introduce the local dynamo number

$$
\mathcal{D}=D \alpha_{1}(r) S(r),
$$

a function of galactocentric radius that quantifies the dynamo efficiency at a given $r$. For a magnetic field growing at an exponential growth rate $\gamma$, i.e., $\partial B_{\phi} / \partial t=\gamma B_{\phi}$ and $\partial A_{\phi} / \partial t=\gamma A_{\phi}$, estimates similar to those presented above yield $\gamma \simeq \sqrt{-\pi \mathcal{D} / 2}-\sqrt{-\pi D_{\mathrm{c}} / 2}$, or in dimensional variables, the local dynamo amplification (e-folding) time is estimated, for $k \epsilon=\pi / 2$, as

$$
\gamma^{-1} \simeq \frac{3 h_{0}^{2}}{l_{0} v_{0}} \frac{1}{3 \epsilon\left(V_{0} / v_{0}\right) \sqrt{-\pi S \Omega /\left(2 \Omega_{0}^{2}\right)}-\pi^{2} / 2} \simeq 10^{9} \mathrm{yr},
$$


where the numerical value is obtained for $\epsilon=0.5, V_{0} / v_{0}=3, v_{0}=10 \mathrm{~km} \mathrm{~s}^{-1}, h_{0}=0.5 \mathrm{kpc}, l_{0} / h_{0}=0.2$ and $S \Omega / \Omega_{0}^{2}=-1$.

The ratio $D / D_{\mathrm{c}}$ and $\gamma$ are maximal, whereas $V_{\mathrm{c}}$ is the minimum, for the mode with the radial wave number $k=2 /(\pi \epsilon)$. For this leading mode, we obtain $D / D_{\mathrm{c}} \simeq\left(9 / \pi^{3}\right) \epsilon^{2}\left(V_{0} / v_{0}\right)^{2}$ and $V_{\mathrm{c}} \simeq \pi^{2} v_{0} /(6 \epsilon) \simeq$ $3 v_{0} \simeq 30 \mathrm{~km} \mathrm{~s}^{-1}$, with the numerical value obtained for $\epsilon=0.5$.

These estimates implicitly assume the quadrupolar parity of the large-scale magnetic field since the relations like $\left|\partial B_{\phi} / \partial z\right| \simeq \pi\left|B_{\phi}\right| / 2,\left|\partial^{2} B_{\phi} / \partial z^{2}\right| \simeq-\pi^{2}\left|B_{\phi}\right| / 4$ and $\left|\mathcal{L}_{r}\left(B_{\phi}\right)\right| \simeq-k^{2}\left|B_{\phi}\right|$ only apply in this case. The asymptotic solution for $|D| \ll 1$ [46] (not presented here) shows that dipolar modes have much higher values of $\left|D_{\mathrm{c}}\right|$ and $V_{\mathrm{c}}$ and are oscillatory.

\section{Numerical Solutions}

We confirmed those estimates using numerical solutions of the dynamo equation in a thick disc. Equations (8) and (9) were solved within a rectangle $(0,0) \leq(r, z) \leq\left(R_{\mathrm{b}}, h_{0}\right)$ in the $(r, z)$-plane. We adopted $R_{\mathrm{b}}=4$ and verified that the results were not sensitive to the position of the outer radial boundary when $R_{\mathrm{b}}=3-10$. The boundary conditions adopted in radius, $\partial\left(r B_{\phi}\right) / \partial r=\partial\left(r A_{\phi}\right) / \partial r=0$ at $r=R_{\mathrm{b}}$ correspond to vanishing diffusive flux of magnetic field. The boundary conditions on the disc surface, $B_{\phi}=\partial A_{\phi} / \partial z=0$ at $|z|=h$, apply when large-scale electric currents outside the disc are much weaker than those within the disc (the vacuum boundary conditions). The boundary condition for $A_{\phi}$ at $|z|=h$ is an approximation to $\partial A_{\phi} / \partial z=\mathcal{O}\left(\epsilon A_{\phi}\right)$, and its accuracy reduces as $\epsilon$ increases ([5] and references therein). Boundary conditions at the disc mid-plane control the symmetry (either quadrupolar/even or dipolar/odd parity) of the magnetic field across the disc (see [1] for details)

$$
\begin{array}{ll}
\left.\frac{\partial B_{\phi}}{\partial z}\right|_{z=0}=\left.A_{\phi}\right|_{z=0}=0 & \text { (quadrupolar), } \\
\left.B_{\phi}\right|_{z=0}=\left.\frac{\partial A_{\phi}}{\partial z}\right|_{z=0}=0 & \text { (dipolar) }
\end{array}
$$

and we are free to consider the range $z>0$ because of the symmetry of the solutions. The initial magnetic field is of a large scale, with sinusoidal variation in both $r$ and $z$ to satisfy the boundary conditions and has the average strength $|\boldsymbol{B}| \approx 10^{-3}$ in dimensionless units (its magnitude does not, in fact, matter as long as kinematic dynamo solutions are considered).

The solutions were obtained for the rotation curve (4) and $\alpha(r, z)=\Omega(r) \sin (\pi z)$ in dimensionless units. Forward time stepping was performed using the fourth-order Runge-Kutta scheme and central finite differences were used for the spatial derivatives. The consistency of the results was verified by increasing the grid resolution. Here, we present results for $64 \times 64$ and $128 \times 128$ grids, with a uniform spacing in both radial and vertical directions (in terms of dimensionless variables).

An efficient technique [47] to find the critical value of the dynamo number, $D_{\mathrm{c}}$, for a given $\alpha(r, z)$ is to solve the dynamo equations for a sufficiently large (supercritical) $R_{\alpha}$ with a modified form of the coefficient, $\hat{\alpha}(r, z)$, that depends on $|\boldsymbol{B}|$ (so that the field is eventually driven to a steady state) but has the same spatial form as $\alpha(r, z)$ at all times:

$$
\widehat{\alpha}(r, z, t)=\frac{\alpha_{0} \alpha(r, z)}{1+2 \pi W^{-1} \int_{0}^{R_{\mathrm{b}}} r \mathrm{~d} r \int_{0}^{h} \mathrm{~d} z\left|\boldsymbol{B}(r, z, t) / B_{0}\right|^{2}},
$$

where $W=\pi h R_{\mathrm{b}}^{2}$ is the volume of the computational domain, and we used dimensional variables. The value of $\widehat{\alpha}(r, z, t) / \alpha(r, z)$ in the steady state (when $\widehat{\alpha}$ no longer varies with $t$ ) for $\alpha_{0}$ in Equations (10) and (12) yields $D_{\mathrm{c}}$. 


\section{Results}

Our goal was to clarify conditions required for mean-field dynamo action in the thick discs of low-mass galaxies rather than to explore magnetic field forms and distributions in specific galaxies. Therefore, we focused on finding the critical values of the dynamo number and the corresponding rotational speed and only briefly discuss steady-state magnetic field distributions.

\subsection{Critical Rotation Speed}

We solved Equations (8) and (9) with the vacuum boundary conditions and symmetry conditions (19) for a range of the disc aspect ratios $\epsilon$ to obtain $D_{c}$, and then the corresponding critical rotation speed was obtained, which was then compared with Equation (16). For given $R_{0}$, the disc aspect ratio $\epsilon$ is varied by changing the characteristic disc scale height $h_{0}$. We recall that the large-scale magnetic field can be maintained if $|D| \geq\left|D_{\mathrm{c}}\right|$, or, equivalently, $V_{0} \geq V_{\mathrm{c}}$, and decays otherwise. Figure 1 shows $V_{\mathrm{c}}$ as a function of $\epsilon$; Figure 2 presents the variation of $D_{\mathrm{c}}$. As expected, weaker rotation is required to excite the dynamo as the disc becomes thicker.

It is remarkable how accurately Equations (14) and (16) reproduce the variations of $D_{\mathrm{c}}$ and $V_{\mathrm{c}}$ with $\epsilon$. The best agreement between the approximate solution of Section 3 and the numerical results was achieved for $k^{2} \approx 8$. This value of $k$ is of the order of unity, as expected, but it still exceeds unity, which indicates that the magnetic field has the radial scale of order $R_{0} / k$, about a third of the disc radius, in qualitative similarity to the kinematic dynamo solutions in a thin disc [1]. The parameter $k$ is akin to the radial wave number and its inverse is a measure of the radial scale of the magnetic field compared to the characteristic length scale $R_{0}$.

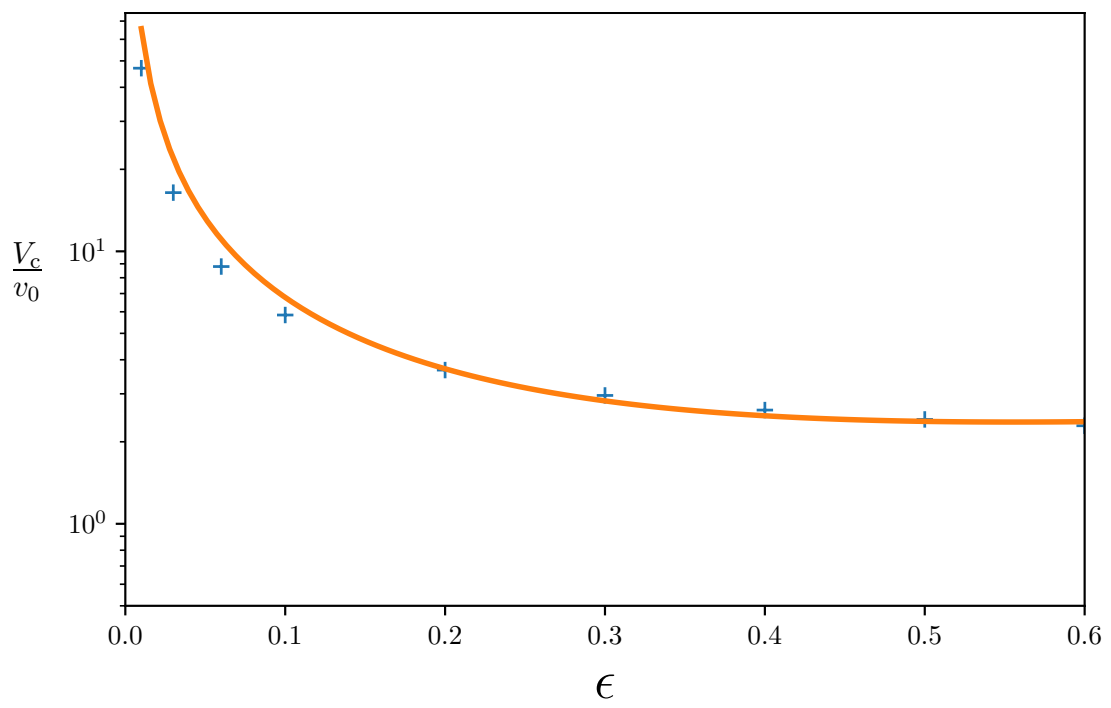

Figure 1. The variation of the critical rotation speed $V_{\mathrm{c}}$ required for the dynamo action (for the quadrupolar magnetic field geometry) with the disc aspect ratio $\epsilon$ : numerical results are shown with crosses and the solid line represents the approximate value from Equation (16) with $k^{2}=8$. 


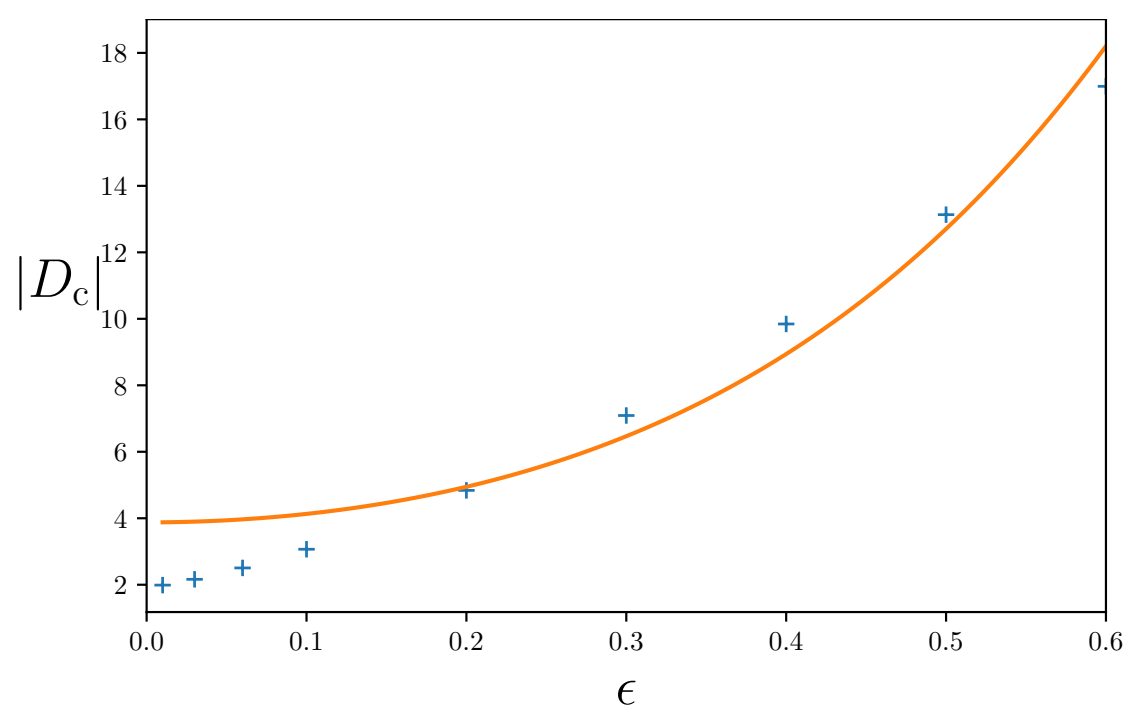

Figure 2. As in Figure 1 but for the critical dynamo number, Equation (15).

Figure 1 confirms that even weak rotation, with the maximum rotation speed only a few times larger than the turbulent speed, is sufficient to support the mean-field dynamo action in a thick disc typical of a low-mass galaxy. For the aspect ratios close to $\epsilon=0.3-0.5$, even $V_{0} \simeq 20-30 \mathrm{~km} \mathrm{~s}^{-1}$ appears to be be sufficient to maintain a large-scale quadrupolar magnetic field. For a dipolar field in a disc of a similar aspect ratio, the critical velocity is about $100 \mathrm{~km} \mathrm{~s}^{-1}$.

\subsection{Magnetic Field Geometry}

Large-scale magnetic fields of quadrupolar parity dominate in thin discs [1] as their critical dynamo number is lower than that for a dipolar magnetic field (or, equivalently, the magnetic field growth rate is higher for quadrupolar parity for a given dynamo number), and, as shown here, this difference is maintained in a thicker layer although it decreases as the disc becomes thicker ( $\epsilon$ increases). In a sphere, dipolar and quadrupolar modes are generated as almost equal ease [48].

The structure of magnetic field obtained for $D=D_{\mathrm{c}}$ is shown in Figures 3-5. Typically of the eigenfunctions of the kinematic dynamo problem, magnetic field is localised near the maximum of the rotational shear [49]. Magnetic pitch angles shown in Figures $3 \mathrm{~d}$ and $4 \mathrm{~d}$ are similar to those observed in galaxies, $-\left(10-30^{\circ}\right)$, with negative values indicative of a spiral trailing with respect to the galactic rotation. Since the rotational shear in low-mass galaxies is lower than in massive spiral galaxies, the magnitude of the pitch angle is larger in the former; i.e., magnetic spirals are less tightly wound. Nonlinear dynamo effects, neglected here, lead to the saturation of the exponential growth of the magnetic field and to its spread along the disc. We do not discuss such nonlinear solutions here because they are more relevant in more realistic dynamo models that allow, in particular, for deviations of the discs of low-mass galaxies from axial symmetry. 

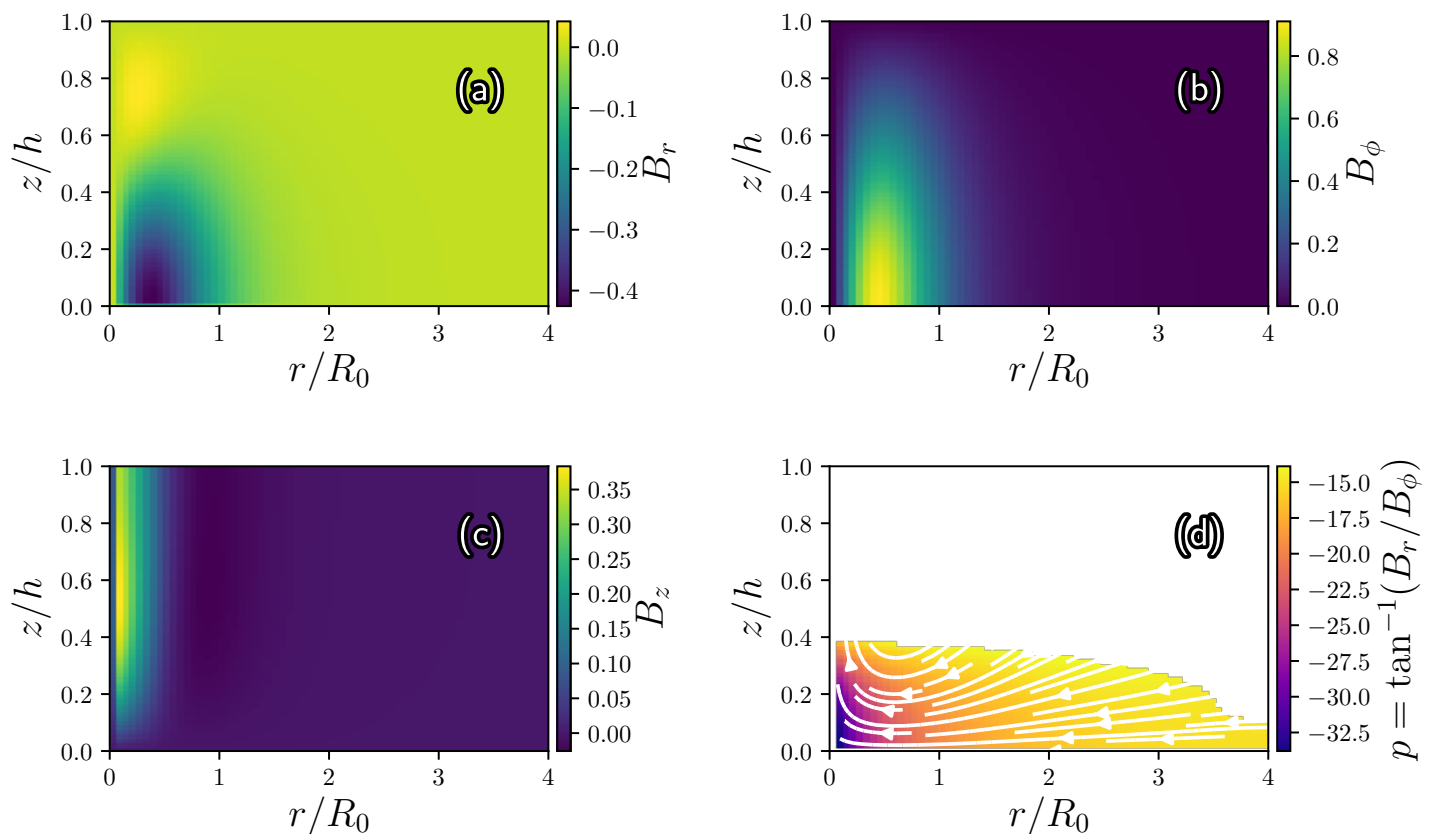

Figure 3. The cylindrical components (a) $B_{r}$, (b) $B_{\phi}$, and (c) $B_{z}$ of the marginally stable quadrupolar eigenfunction of the $\alpha \omega$-dynamo equations for $R_{0}=1 \mathrm{kpc}, V_{0} / v_{0}=2.96$, and $\epsilon=0.3$ normalised to $\max \left(B_{r}^{2}+B_{z}^{2}+B_{\phi}^{2}\right)=1$. Panel (d) shows poloidal magnetic lines (white), providing an indication of the relationship between $B_{z}$ and $B_{r}$, and the pitch angle of magnetic lines (in degrees, colour). Only the region where $B_{r}^{2}+B_{z}^{2}+B_{\phi}^{2} \geq 0.01 \max \left(B_{r}^{2}+B_{z}^{2}+B_{\phi}^{2}\right)$ is shown.
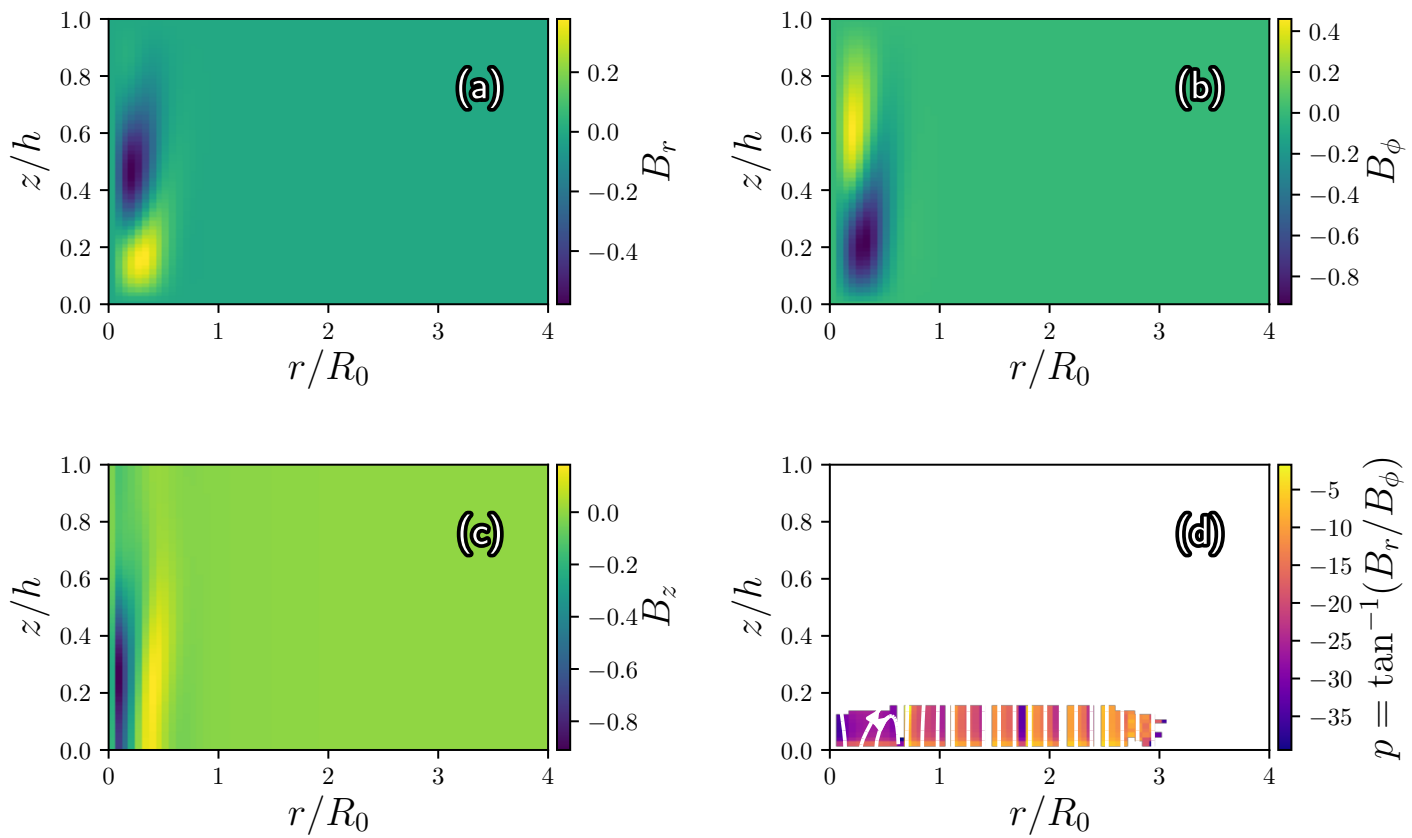

Figure 4. As in Figure 3 but for the dipolar parity. The critical circular rotation speed $V_{\mathrm{c}} / v_{0}=17$ is higher than for the marginal quadrupolar mode. Unlike the quadrupolar field of Figure 3, the dipolar magnetic field varies periodically in time (see Figure 5). 

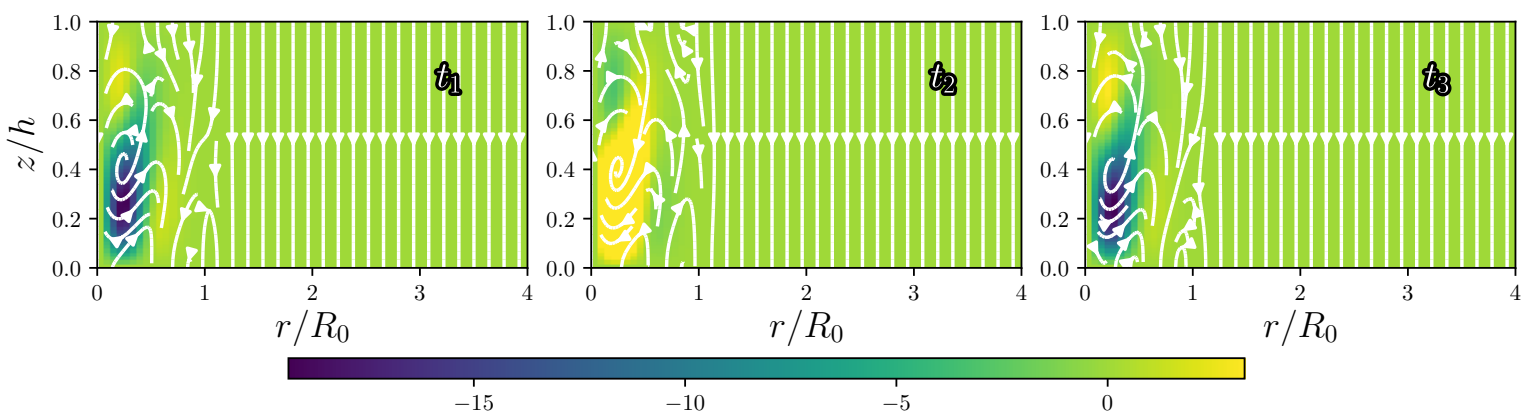

Figure 5. The form of the marginally stable dipolar mode of Figure 4 in various phases of its oscillation: magnetic lines of the poloidal field are shown white and the toroidal component is colour-coded (arbitrary units) for three different times within the oscillation period $\left(t_{1}<t_{2}<t_{3}\right)$.

\section{Discussion and Conclusions}

Low-mass galaxies rotate slower than massive disc galaxies, and hence the driving of the dynamo due to both rotational velocity shear and the $\alpha$-effect is weaker. The intensity of interstellar turbulence in low-mass galaxies is not lower than in massive disc galaxies, so the turbulent magnetic diffusivity which destroys the mean magnetic field is similar in both massive and low-mass galaxies. We have shown that the ratio of the disc thickness to its radius plays a crucial role: a mean-field dynamo can amplify and maintain magnetic fields in thicker discs at smaller rotational speeds than in thin discs because, for a fixed disc radius, the turbulent diffusion time across the disc is longer in a thicker layer, and hence large-scale magnetic fields require lower rotational velocity shear to be maintained. Approximate solutions of kinematic $\alpha \omega$-dynamo equations applicable to thick discs obtained here clearly demonstrate an increase in the dynamo efficiency as the disc aspect ratio increases. The accuracy of the approximate solution was confirmed using numerical solutions of the dynamo equations. We conclude that mean-field dynamo can produce large-scale magnetic fields in low-mass galaxies.

We have only considered axially symmetric solutions of the kinematic dynamo equations. Those are the main simplifications adopted in this work but they are appropriate when the origin of magnetic fields is considered rather than their form in specific galaxies. The discs of low-mass galaxies often exhibit strong deviations from axial symmetry, and this would affect the large-scale magnetic field. There are no reasons to expect that the mean-field dynamo action in a thick axisymmetric disc might support non-axisymmetric dynamo modes: they are difficult to maintain even in a thin disc, and the difficulty increases as the disc becomes thicker [1]. Therefore, the global magnetic structures observed in low-mass galaxies can be considered as a result of the distortion of a basic axially symmetric magnetic field by the deviations of the galaxy from axial asymmetry. Applications of dynamo theory to specific galaxies with allowance for deviations from axial symmetry and for the nonlinear saturation of the dynamo action will be discussed elsewhere.

Author Contributions: conceptualization and methodology, A.S. and K.S.; software and analysis, P.B.; writing-review and editing, P.B., A.S., and K.S.

Funding: A.S. acknowledges financial support of the STFC (ST/N000900/1, Project 2) and the Leverhulme Trust (RPG-2014-427).

Acknowledgments: A.S. expresses gratitude to IUCAA for hospitality and financial support. K.S. is grateful to School of Mathematics, Statistics, and Physics of Newcastle University for hospitality. P.B. thanks Arunima Benerjee, Eric Blackman, Luke Chamandy, Jayaram Chengalur, Dipanjan Mitra, and Subhashis Roy for helpful comments. We also thank the reviewers for valuable suggestions. 
Conflicts of Interest: The authors declare no conflict of interest. The funders had no role in the design of the study; in the collection, analyses, or interpretation of data; in the writing of the manuscript, or in the decision to publish the results.

\section{rences}

1. Ruzmaikin, A.; Shukurov, A.; Sokoloff, D. Magnetic Fields of Galaxies; Kluwer: Dordrecht, The Netherlands, 1988. doi:10.1007/978-94-009-2835-0. [CrossRef]

2. Beck, R.; Brandenburg, A.; Moss, D.; Shukurov, A.; Sokoloff, D. Galactic magnetism: Recent developments and perspectives. Ann. Rev. Astron. Astrophys. 1996, 34, 155-206. doi:10.1146/annurev.astro.34.1.155. [CrossRef]

3. Beck, R. Magnetic fields in spiral galaxies. Astron. Astrophys. Rev. 2015, 24, 4. doi:10.1007/s00159-015-0084-4. [CrossRef]

4. Brandenburg, A.; Subramanian, K. Astrophysical magnetic fields and nonlinear dynamo theory. Phys. Rep. 2005, 417, 1-209, doi:10.1016/j.physrep.2005.06.005. [CrossRef]

5. Shukurov, A. Galactic dynamos. In Mathematical Aspects of Natural Dynamos; Dormy, E., Soward, A.M., Eds.; Chapman \& Hall/CRC: New York, NY, USA: 2007; pp. 313-359.

6. Chamandy, L.; Shukurov, A.; Subramanian, K.; Stoker, K. Non-linear galactic dynamos: A toolbox. Mon. Not. R. Astron. Soc. 2014, 443, 1867-1880. doi:10.1093/mnras/stu1274. [CrossRef]

7. Shukurov, A.; Subramanian, K. Astrophysical Magnetic Fields: From Galaxies to the Early Universe; CUP: Cambridge, UK, 2020.

8. Beck, R.; Fletcher, A.; Shukurov, A.; Snodin, A.; Sokoloff, D.D.; Ehle, M.; Moss, D.; Shoutenkov, V. Magnetic fields in barred galaxies. IV. NGC 1097 and NGC 1365. Astron. Astrophys. 2005, 444, 739-765, doi:10.1051/0004-6361:20053556. [CrossRef]

9. Van Eck, C.L.; Brown, J.C.; Shukurov, A.; Fletcher, A. Magnetic fields in a sample of nearby spiral galaxies. Astrophys. J. 2015, 799, 35. doi:10.1088/0004-637X/799/1/35. [CrossRef]

10. Chamandy, L.; Shukurov, A.; Taylor, A.R. Statistical tests of galactic dynamo theory. Astrophys. J. $2016,833,43$. doi:10.3847/1538-4357/833/1/43. [CrossRef]

11. Rodrigues, L.F.S.; Shukurov, A.; Fletcher, A.; Baugh, C.M. Galactic magnetic fields and hierarchical galaxy formation. Mon. Not. R. Astron. Soc. 2015, 450, 3472-3489. doi:10.1093/mnras/stv816. [CrossRef]

12. Rodrigues, L.F.S.; Chamandy, L.; Shukurov, A.; Baugh, C.M.; Taylor, A.R. Evolution of galactic magnetic fields. Mon. Not. R. Astron. Soc. 2019, 483, 2424-2440. doi:10.1093/mnras/sty3270. [CrossRef]

13. Chyży, K.T.; Beck, R.; Kohle, S.; Klein, U.; Urbanik, M. Regular magnetic fields in the dwarf irregular galaxy NGC 4449. Astron. Astrophys. 2000, 355, 128-137.

14. Haynes, R.F.; Klein, U.; Wayte, S.R.; Wielebinski, R.; Murray, J.D.; Bajaja, E.; Meinert, D.; Buczilowski, U.R.; Harnett, J.I.; Hunt, A.J.; et al. A radio continuum study of the Magellanic Clouds. I. Complete multi-frequency maps. Astron. Astrophys. 1991, 252, 475-486.

15. Mao, S.A.; Gaensler, B.M.; Stanimirović, S.; Haverkorn, M.; McClure-Griffiths, N.M.; Staveley- Smith, L.; Dickey, J.M. A radio and optical polarization study of the magnetic field in the Small Magellanic Cloud. Astrophys. J. 2008, 688, 1029-1049. doi:10.1086/590546. [CrossRef]

16. Mao, S.A.; McClure-Griffiths, N.M.; Gaensler, B.M.; Haverkorn, M.; Beck, R.; McConnell, D.; Wolleben, M.; Stanimirović, S.; Dickey, J.M.; Staveley-Smith, L. Magnetic field structure of the Large Magellanic Cloud from Faraday rotation measures of diffuse polarized emission. Astrophys. J. 2012, $759,25$. doi:10.1088/0004-637X/759/1/25. [CrossRef]

17. Kepley, A.A.; Mühle, S.; Everett, J.; Zweibel, E.G.; Wilcots, E.M.; Klein, U. The role of the magnetic field in the interstellar medium of the post-starburst dwarf irregular galaxy NGC 1569. Astrophys. J. 2010, 712, 536-557. doi:10.1088/0004-637X/712/1/536. [CrossRef]

18. Chyży, K.T.; Weżgowiec, M.; Beck, R.; Bomans, D.J. Magnetic fields in Local Group dwarf irregulars. Astron. Astrophys. 2011, 529, A94. doi:10.1051/0004-6361/201015393. [CrossRef] 
19. Hindson, L.; Kitchener, G.; Brinks, E.; Heesen, V.; Westcott, J.; Hunter, D.; Zhang, H.X.; Rupen, M.; Rau, U. A radio continuum study of dwarf galaxies: $6 \mathrm{~cm}$ imaging of LITTLE THINGS. Astrophys. J. Suppl. Ser. 2018, $234,29$. doi:10.3847/1538-4365/aaa42c. [CrossRef]

20. Beck, R.; Wielebinski, R. Magnetic fields in galaxies. In Planets, Stars and Stellar Systems; Oswalt, T.D., Gilmore, G., Eds.; Springer: Dordrecht, The Netherlands, 2013; Volume 5, p. 641. doi:10.1007/978-94-007-5612-0_13. [CrossRef]

21. Walter, F.; Brinks, E. Holes and shells in the interstellar medium of the nearby dwarf galaxy IC 2574. Astron. J. 1999, 118, 273-301. doi:10.1086/300906. [CrossRef]

22. Wilcots, E.M.; Miller, B.W. The kinematics and distribution of H I in IC 10. Astron. J. 1998, 116, $2363-2394$. doi:10.1086/300595. [CrossRef]

23. Oh, S.H.; Hunter, D.A.; Brinks, E.; Elmegreen, B.G.; Schruba, A.; Walter, F.; Rupen, M.P.; Young, L.M.; Simpson, C.E.; Johnson, M.C.; et al. High-resolution mass models of dwarf galaxies from LITTLE THINGS. Astron. J. 2015, 149, 180. doi:10.1088/0004-6256/149/6/180. [CrossRef]

24. Iorio, G.; Fraternali, F.; Nipoti, C.; Di Teodoro, E.; Read, J.I.; Battaglia, G. LITTLE THINGS in 3D: Robust determination of the circular velocity of dwarf irregular galaxies. Mon. Not. R. Astron. Soc. 2017, 466, 4159-4192. doi:10.1093/mnras/stw3285. [CrossRef]

25. Martimbeau, N.; Carignan, C.; Roy, J.R. Dark matter distribution and the H I-H $\alpha$ connection in IC 2574. Astron. J. 1994, 107, 543-554. doi:10.1086/116875. [CrossRef]

26. Staveley-Smith, L.; Davies, R.D.; Kinman, T.D. H I and optical observations of dwarf galaxies. Mon. Not. R. Astron. Soc. 1992, 258, 334-346. doi:10.1093/mnras/258.2.334. [CrossRef]

27. Roychowdhury, S.; Chengalur, J.N.; Karachentsev, I.D.; Kaisina, E.I. The intrinsic shapes of dwarf irregular galaxies. Mon. Not. R. Astron. Soc. 2013, 436, L104-L108. doi:10.1093/mnrasl/slt123. [CrossRef]

28. Banerjee, A.; Jog, C.J.; Brinks, E.; Bagetakos, I. Theoretical determination of H I vertical scale heights in the dwarf galaxies DDO 154, Ho II, IC 2574 and NGC 2366. Mon. Not. R. Astron. Soc. 2011, 415, 687-694. doi:10.1111/j.1365-2966.2011.18745.x. [CrossRef]

29. Zeldovich, Y.B.; Ruzmaikin, A.A.; Sokoloff, D.D. The Almighty Chance; World Scientific: Singapore, 1990. doi:10.1142/0862. [CrossRef]

30. Moss, D.; Sokoloff, D. Galactic winds and the origin of large-scale magnetic fields. Astron. Astrophys. 2017, 598, A72, doi:10.1051/0004-6361/201628893. [CrossRef]

31. Ivers, D.J. Kinematic dynamos in spheroidal geometries. Proc. Roy. Soc. Lond. Ser. A $2017,473$. doi:10.1098/rspa.2017.0432. [CrossRef]

32. Stix, M. The galactic dynamo. Astron. Astrophys. 1975, 42, 85-89.

33. Brandenburg, A.; Tuominen, I.; Krause, F. Dynamos with a flat $\alpha$-effect distribution. Geophys. Astrophys. Fluid Dyn. 1990, 50, 95-112. doi:10.1080/03091929008219875. [CrossRef]

34. Elstner, D.; Meinel, R.; Rüdiger, G. Galactic dynamo models without sharp boundaries. Geophys. Astrophys. Fluid Dyn. 1990, 50, 85-94. doi:10.1080/03091929008219874. [CrossRef]

35. Siejkowski, H.; Soida, M.; Chyży, K.T. Magnetic field evolution in dwarf and Magellanic-type galaxies. Astron. Astrophys. 2018, 611, A7. doi:10.1051/0004-6361/201730566. [CrossRef]

36. Siejkowski, H.; Soida, M.; Otmianowska-Mazur, K.; Hanasz, M.; Bomans, D.J. Cosmic-ray driven dynamo in the interstellar medium of irregular galaxies. Astron. Astrophys. 2010, 510, A97. doi:10.1051/0004-6361/200912729. [CrossRef]

37. Siejkowski, H.; Otmianowska-Mazur, K.; Soida, M.; Bomans, D.J.; Hanasz, M. 3D global simulations of a cosmic-ray-driven dynamo in dwarf galaxies. Astron. Astrophys. 2014, 562, A136. doi:10.1051/0004-6361/201220367. [CrossRef]

38. Moffatt, H.K. Magnetic Field Generation in Electrically Conducting Fluids; CUP: Cambridge, UK, 1978.

39. Parker, E.N. Cosmical Magnetic Fields. Their Origin and Their Activity; Clarendon Press: Oxford, UK, 1979.

40. Krause, F.; Rädler, K.H. Mean-field Magnetohydrodynamics and Dynamo Theory; Pergamon Press: Oxford, UK, 1980.

41. Zeldovich, Y.B.; Ruzmaikin, A.A.; Sokoloff, D.D. Magnetic Fields in Astrophysics; Gordon and Breach: New York, NY, USA, 1983. 
42. Shukurov, A.; Rodrigues, L.F.S.; Bushby, P.J.; Hollins, J.; Rachen, J.P. A physical approach to modelling large-scale galactic magnetic fields. Astron. Astrophys. 2019, 623, A113, doi:10.1051/0004-6361/201834642. [CrossRef]

43. Subramanian, K.; Mestel, L. Galactic dynamos and density wave theory - II. An alternative treatment for strong non-axisymmetry. Mon. Not. R. Astron. Soc. 1993, 265, 649-654. doi:10.1093/mnras/265.3.649. [CrossRef]

44. Phillips, A. A comparison of the asymptotic and no-z approximations for galactic dynamos. Geophys. Astrophys. Fluid Dyn. 2001, 94, 135-150. doi:10.1080/03091920108204133. [CrossRef]

45. Ji, Y.; Cole, L.; Bushby, P.; Shukurov, A. Asymptotic solutions for mean-field slab dynamos. Geophys. Astrophys. Fluid Dyn. 2014, 108, 568-583. doi:10.1080/03091929.2014.898757. [CrossRef]

46. Shukurov, A.; Sokoloff, D. Astrophysical dynamos. In Dynamos: Lecture Notes of the Les Houches Summer School 2007; Cardin, P., Cugliandolo, L., Eds.; Elsevier Science: Amsterdam, The Netherlands, 2008; pp. 251-299.

47. Rädler, K.H.; Wiedemann, E. Numerical experiments with a simple nonlinear mean-field dynamo model. Geophys. Astrophys. Fluid Dyn. 1989, 49, 71-79. doi:10.1080/03091928908243464. [CrossRef]

48. Proctor, M.R.E. On the eigenvalues of kinematic $\alpha$-effect dynamos. Astron. Nachr. $1977,298,19$. doi:10.1002/asna.19772980104. [CrossRef]

49. Ruzmaikin, A.; Shukurov, A.; Sokoloff, D.; Starchenko, S. Maximally-efficient-generation approach in the dynamo theory. Geophys. Astrophys. Fluid Dyn. 1990, 52, 125-139. doi:10.1080/03091929008219843. [CrossRef]

(C) 2019 by the authors. Licensee MDPI, Basel, Switzerland. This article is an open access article distributed under the terms and conditions of the Creative Commons Attribution (CC BY) license (http:/ / creativecommons.org/licenses/by/4.0/). 